\title{
La investigación aplicada a los estudios de mercado
}

\section{La investigación aplicada a los estudios de mercado}

\author{
Luis Antonio Tobar \\ Sociólogo e investigador de la Facultad Multidisciplinaria de Occidente \\ Universidad de El Salvador \\ tobarquintero90@gmail.com
}

Fecha de recepción: 12 de febrero de 2019 Fecha aprobación: 10 de noviembre de 2019 DOI: $10.5377 /$ ryr.v50i50.9040

\section{RESUMEN}

Los estudios sobre la investigación de mercados se desarrollaron en un principio por expertos en Ciencias Sociales, los cuales al llevar a cabo estudios de opinión pública fueron tomados en cuenta para los mismos. La investigación como un componente fundamental en cualquier área resulta muy importante para realizar una variedad de estudios sobre el consumo, preferencias por marcas, entre otros. Los estudios de mercado toman importancia a tal grado, que hoy en día existen departamentos académicos en las principales universidades del mundo, utilizando los métodos cualitativos y cuantitativos para levar a cabo este tipo de estudios.

Palabras clave: ciencia, investigación, estudios de mercado, cualitativo, cuantitativo, técnicas de investigación.

\section{ABSTRACT}

The studies about market researches in which it was taken into account the development of public opinion researches were at beginning developed by experts in social sciences. The research as a fundamental component in any área results very important in order to develop a variety of studies about consumism, label preferences, and so forth. The market researches take important in such level that now days there exists academic departments in the main worldwide universities, using qualitative and quantitative methods to develop this type of researches.

Keywords: science, research, market researches, qualitative, quantitative, research techniques. 


\section{Introducción}

La investigación es uno de los elementos, dentro del mundo académico, más importante para estudiar la realidad social. La ciencia a través de la técnica se auxilia de procesos de investigación para conocer, de manera general, los fenómenos de las ciencias naturales y sociales, el tema aquí estudiado es vital para una ciencia social como la mercadotecnia.

El objetivo de este artículo es establecer la relación que se presenta entre los métodos y técnicas de investigación y los estudios de mercado, vitales para entender las preferencias de consumo por algunos estratos sociales y el porqué prefieren ese tipo de productos. La investigación de mercados tomó auge en los últimos cincuenta años del siglo $\mathrm{XX}$, debido al profundo crecimiento de los mercados a nivel mundial. Desarrollado por las universidades en sus principios y después en las empresas transnacionales como un instrumento para tomar decisiones a la hora de lanzar sus productos.

En los últimos años, es común encontrar estudios de mercado en todo tipo de empresas y cátedras en las universidades, por ello, muchas de las investigaciones realizadas en la publicidad y mercadotecnia han girado en este aspecto de la economía, que representa uno de los intereses de mayor peso en las empresas. Las siguientes líneas profundizan en estos aspectos y su relación con la investigación y la ciencia.

\section{La ciencia y el desarrollo de la investigación}

El desarrollo de la ciencia a través de la historia ha sido de mucho valor para resolver los problemas en una determinada sociedad. El conocimiento científico producido por la ciencia genera que los países desarrollen investigación en diferentes ramas. El establecimiento de leyes generales permite construir una serie de categorías y conceptos que buscan indagar en la producción de conocimientos científicos, para la solución de diferentes fenómenos en las sociedades.

Asimismo, hay una utilización de diferentes métodos, mediante los cuales la investigación científica busca interpretar la realidad en las diferentes ciencias. Además, la generación de hipótesis, a través de los resultados, busca llegar a conclusiones sólidas sobre una serie de problemas que afectan a los seres humanos en su vida cotidiana. Por ello, Kédrov y Spirkin (1968) plantean:

(...) La ciencia es un sistema de conceptos acerca de los fenómenos y leyes del mundo externo o de la actividad espiritual de los individuos, que permite prever y transformar la realidad en beneficio de la sociedad; una forma de actividad humana históricamente establecida, una producción espiritual, cuyo contenido y resultado es la reunión de hechos orientados en un determinado sentido, de hipótesis y teorías elaboradas y de las leyes que constituyen su fundamento, así como de procedimientos y métodos de investigación (p. 7).

La actividad científica está dirigida a formular leyes e hipótesis sobre distintos fenómenos de la naturaleza y la sociedad. En el transcurso de la historia, la ciencia ha evolucionado y se ha perfeccionado mediante el avance de la técnica; es decir, el conocimiento científico 
ha sido perfeccionado mediante la actividad trasformadora de la naturaleza. Los primeros conocimientos aparecieron en el hombre primitivo, los cuales se originaron en la conciencia y el establecimiento de relaciones sociales con otros.

En ese sentido, Spirkin (1968) menciona que cada hombre llega al conocimiento de manera individual, por medio de sus sentidos y el cerebro, pero el cual no puede generarse de una manera extrasocial, sin establecerse relaciones con los otros hombres. Dicho conocimiento en sus primeros estadios, se trató de explicar desde lo cotidiano intentando conocer cómo apareció tal fenómeno, mientras que con el avance de los años se desarrolló el científico, que daba respuesta del porqué aparecían ciertos hechos.

El objeto de la ciencia puede caracterizarse en dos grandes ramas: las generales y las particulares. Las primeras abarcan todas aquellas ciencias que establecen postulados generales de la realidad, por ejemplo, la filosofía y lógica, mientras que las segundas son las que se dedican al estudio de fenómenos particulares, tales como la química, la mecánica, psicología, derecho, etc.

Así pues, para estudiar las diferentes ciencias se utilizan métodos e instrumentos que permiten comprobar los fenómenos que estudian dichas ramas. Kedrov y Spirkin (1968) plantean: “Los medios que se utilizan en la investigación científica son los aparatos, instrumentos, etc., que sirven para estudiar y comprobar experimentalmente el objeto en cuestión y también para fijar y elaborar los resultados obtenidos (...)" (p. 17).
Las ideas anteriores parten de la existencia de un método que permite comprobar los fenómenos, según el objeto de cada ciencia, lo que lleve al establecimiento de conceptos, categorías y leyes. Además, de producir conocimientos sobre nuevos problemas ocurridos en la naturaleza y la sociedad, los cuales necesitan una respuesta certera para los investigadores. De tal manera, que el investigar tiene la tarea, en palabras de Dieterich (1996), de producir nuevos conocimientos objetivos sobre la realidad.

Para llevar a cabo esta tarea debe cumplir tres requisitos:

a. La persona que investiga debe estar dispuesta a utilizar el método y la ética científica.

b. Debe tener preparación metodológica para su empleo correcto.

c. Debe contar con los recursos necesarios para llevar la indagación a buen término.

Los requisitos anteriores muestran que el camino de la producción científica necesita el desarrollo de la técnica como medio fundamental para la solución de distintos problemas. Por ende, el desarrollo de la ciencia está determinada por el incremento de los bienes de producción, lo cual obliga a los científicos a buscar nuevos métodos y técnicas que mejoren los niveles de productividad de una sociedad.

Así en el siglo XVIII, las ciencias naturales comenzaron a presentar un notable desarrollo, el nuevo modo de producción necesitaba que las mercancías se produjeran más rápido. La química, física, mecánica, economía y medicina 
fueron algunas de las ciencias con más desarrollo de la época. Este nuevo escenario produjo que las pruebas experimentales, ya no se dieran de manera aislada sino en grandes laboratorios, la industria tomó una nueva importancia y la puesta en el mercado de nuevos productos se hizo necesario. Por lo tanto, Navas (2014), menciona:

El hombre se relaciona con la naturaleza, de la que es parte, y a las que, haciendo uso de la racionalidad y de su libertad, se propone y se esfuerza por controlarla y por satisfacer su curiosidad y ansia de conocerla cada vez más (p. 4).

\section{Los métodos y las técnicas en la investigación}

De manera general, Rojas (1996) destaca que, los métodos y las técnicas de investigación sirven para alcanzar los objetivos propuestos mediante un proceso sistemático. Por ello, menciona:

"los métodos y técnicas son las herramientas metodológicas de la investigación, ya que permiten implementar las distintas etapas de estar dirigiendo los procesos mentales y las actividades prácticas hacia la consecución de los objetivos formulados" (p. 64).

Estas ideas implican articular una serie de procesos ordenados y sistematizados que permiten explicar los fenómenos, tomando en cuenta cada una de las características de los métodos de investigación. Utilizados como herramientas, estos permiten interpretar la realidad, aplicando ciertas técnicas. Pero, ¿qué es un método?, una primera definición la plantean Rosental e Iudin (1946): “(...) manera de alcanzar un objetivo, determinado procedimiento para ordenar la actividad (...)" (p. 313). Esto plantea ciertos pasos a seguir para obtener los resultados esperados.

Mientras tanto, Rojas (1996) plantea: “(...) que el método se refiere a criterios y procedimientos generales que guían el trabajo científico para alcanzar un conocimiento objetivo de la realidad (...)" (p. 65). Bajo esta lógica, se partirá a analizar la propuesta de tres enfoques de los métodos de investigación desarrollados en los últimos años, el cualitativo y cuantitativo, tradicionales en la investigación y el pluralismo metodológico crítico de más reciente aparición.

Al estudiar, el método cuantitativo se hace referencia a aquello que tiene relación con datos, población, muestra, hipótesis, entre otros; debido a que su principal apuesta está en comprobar los fenómenos estudiados mediante la formulación de hipótesis. En otras palabras, la medición de los fenómenos es lo que caracteriza a este tipo de investigaciones, por lo cual, su lógica gira en torno a tomar muestras de grandes poblaciones que sean representativas, para que sus resultados sean válidos.

Como lo plantean Danermark, EkstrÖm, Jakobsen y Karlsson (2016), cuando se habla de lo cuantitativo se relaciona con el positivismo y con la matemática, ya que su metodología se basa en el cálculo de fórmulas estadísticas para procesar la información. Dos conceptos importantes para entender lo anterior son la población y la muestra, muy utilizados en dichas investigaciones. La población en palabras de Navas (2014) puede definirse 
como la totalidad de los elementos afectados por el fenómeno a estudiar y que poseen una característica común.

Son ejemplos de población, los siguientes: el número de estudiantes del Centro Escolar INSA; la cantidad de habitantes del departamento de Santa Ana; el total de estudiantes de la Facultad Multidisciplinaria de Occidente de la Universidad de E1 Salvador. Tomando en cuenta a las poblaciones, el segundo paso es sacar una muestra representativa de esta mediante una formula estadística para poblaciones finitas e infinitas. La siguiente figura muestra la diferencia entre una población y la muestra, lo cual ayuda a entender mejor estos dos conceptos en la investigación:
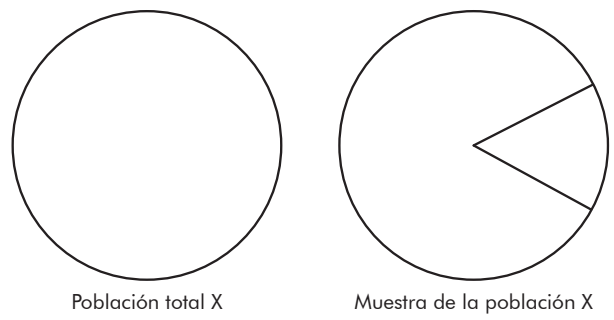

Figura 1. Diferencia entre población y muestra. Fuente: Elaboración propia.

Los resultados que se obtienen, por medio de la investigación cuantitativa, son de carácter general y van de lo particular a lo general. Las conclusiones sobre un determinado fenómeno se generalizan en la totalidad del mismo; es decir, la parte de la muestra que se toma al ser representativa, puede arrojar resultados generalizados. Además, la fórmula utilizada generalmente en las investigaciones de este tipo, es la siguiente:

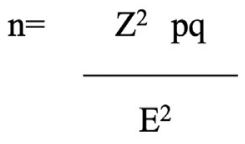

En donde " $Z$ " representa el nivel de confianza requerido.

"pq" se refiere a la variabilidad del fenómeno estudiado.

"E" indica la precisión con que se generalizaran los resultados.

En un segundo momento, se abordará el enfoque cualitativo de la investigación, el cual a diferencia del anterior se centra en las subjetividades del investigador a la hora de estudiar los fenómenos. En palabras de Danermark et al. (2016): "Una característica definitoria dentro de la investigación cualitativa es la focalización en casos individuales (particulares) -uno o varios-. Esto significa que, por ejemplo, se estudia la biografía de una o varias personas, una institución o una sociedad completa (...)" (p. 266). Esta idea explica la verdadera esencia de las investigaciones cualitativas que tienen relación con las subjetividades a la hora de interpretar cualquier fenómeno de la sociedad.

Lo cualitativo está relacionado con la hermenéutica y lo fenomenológico, debido a que los fenómenos sociales deben comprenderse desde un ámbito interpretativo, lo cual no quiere decir que excluye tácitamente lo cuantitativo, sino es una herramienta para analizar la realidad de distinta manera. Las principales técnicas que utiliza este tipo de investigación son: la entrevista en profundidad, la observación participante y no participante, 
estudios de casos, historias de vida, entre otras, que tratan de llegar a los más profundo de la manera de entender los fenómenos.

Para Rojas (1996), "la técnica es un conjunto de reglas y operaciones para el manejo de los instrumentos que auxilia al individuo en la aplicación de los métodos (...)” (p. 65). Estas técnicas son importantes a la hora de obtener la información, por ello es importante que todo investigador, conozca la aplicación correcta de cada una de ella. En los estudios de mercado, las técnicas cualitativas sirven para conocer la preferencia por los productos de los diferentes estratos de consumo.

Como un enfoque complementario a los anteriores, se ha desarrollado el pluralismo metodológico crítico que es la combinación de lo cualitativo y cuantitativo, entendiendo que ambos se complementan a la hora de hacer una investigación. La única diferencia que plantea es que propone estudiar los fenómenos desde una perspectiva epistemológica y ontológica, a través del realismo crítico. Por ende, Bhaskar, citado por Danermark et al. (2016), “(...) el realismo critico implica, dentro de la filosofía, un cambio desde la epistemología hacia la ontología, un cambio desde los acontecimientos hacia los mecanismos (...)" (p. 21).

Lo anterior demuestra que el conocimiento, no solo implica un simple debate entre lo cualitativo y cuantitativo, sino que va más allá de los horizontes superficiales de los fenómenos sociales, tomando en cuenta cómo y por qué surgieron. Esto cambia la perspectiva de la investigación tradicional con la que se venían abordando los problemas dentro de la ciencia. Ello plantea el cómo distintas metodologías llevan a obtener un conocimiento certero, por medio de la utilización de diversos métodos y técnicas.

Así, por ejemplo, los problemas de la ciencia se han venido discutiendo desde la filosofía de los griegos, los cuales dieron pasos importantes para establecer ciertas tendencias que permitieran estudiar la naturaleza y la sociedad. De igual manera, los estudios de Marx profundizaron el estudio de la historia de la economía desde las formaciones precapitalistas para encontrar sus contradicciones. En cuanto a los estudios de mercado, los expertos han tratado de descifrar las preferencias de consumo por diferentes estratos poblacionales que les permitan colocar ciertas mercancías suntuarias.

Este enfoque pretende que los estudios no se detengan en debates superfluos sobre cómo entender los fenómenos, en el sentido de la aplicación de diferentes pasos para estudiar a los individuos. Las preguntas, muestras, resultados, entre otros, solo pueden acercarse a la realidad si muestran visiones epistemológicas y ontológicas de los mismos. Lo cualitativo $y$ cuantitativo son formas de extender el conocimiento de diferentes maneras, que se enriquecen con enfoques teóricos y prácticos que permiten crear un conocimiento más sólido. Danermark et al. (2016) plantean:

El rasgo básico en la realidad social, que de una manera determinante incide en las cuestiones de método, es que la sociedad no puede ser reducida a sus individuos (Weber), tampoco a una totalidad social sin individuos (Durkheim), sino que el mundo está estructurado y estratificado (...) (p.274). 
Estas ideas reflejan lo complicado que significa estudiar los fenómenos sociales, lo cual irá más allá de los debates positivistas y hermenéuticos fenomenológicos. Por lo tanto, la combinación de los enfoques en la investigación es complementario al proponer distintas formas de conocer la realidad.

\section{Lainvestigación aplicada a los estudios de mercado}

Es así como el auge empieza y las universidades empiezan a crear carreras y establecer líneas de investigación de negocios en el interior de las mismas. Pero ¿qué es una investigación de mercados? Benassini (2009) dice que: "La investigación de mercados es la reunión, el registro y el análisis de todos los hechos acerca de los problemas relacionados con las actividades de las personas, las empresas y las instituciones en general (...)" (p. 6)

Cuando aparecen los primeros departamentos dedicados a la investigación de mercados, en palabras de Pope (1984) se organizan en cuatro tipos:

1. Manufactureras. Dupont, General Electric y Kellogg fueron pioneras en el uso de la investigación.

2. Editoriales. Chicago Tribune y Curtis Publishing fueron pioneros.

3. Agencias de publicidad. Lord and Thomas Advertising y N. W. Ayer Advertising son generalmente reconocidas como las primeras agencias en tener funciones de investigación.

4. Universidades. Harvard y Northwestern establecieron cada una, su oficina de investigaciones de negocios antes de 1920 (p. 4).
Posteriormente, este tipo de estudios fue creciendo a medida que la economía mundial crecía y las empresas necesitaban saber, qué tipo de mercancías demandaba el consumidor. Es así como el auge empieza y las universidades empiezan a crear carreras y establecer líneas de investigación de negocios en el interior de las mismas. Pero ¿qué es una investigación de mercados? Benassini (2009) dice que: "La investigación de mercados es la reunión, el registro y el análisis de todos los hechos acerca de los problemas relacionados con las actividades de las personas, las empresas y las instituciones en general (...)" (p. 6).

La internalización del comercio, desarrollo del capitalismo industrial, crecimiento de las economías capitalistas, fueron algunas de las razones que llevaron a las empresas a interesarse por conocer qué tipo de productos se demandaba y el nivel de competitividad de las empresas rivales en el mercado global, para atraer a los clientes mediante la puesta de nuevos productos (desarrollar nuevos productos).

Asimismo, Pilco y Ruiz (2015) establecen que la investigación de mercados nació como una forma estratégica dentro de las empresas, por ende, afirman:

La IM es una disciplina que, mediante un enfoque sistemático y objetivo nos permite recopilar, validar, analizar e interpretar un conjunto de datos con la finalidad de obtener un cierto tipo de información que nos servirá en el proceso de toma de decisiones estratégicas y tácticas en la empresa o institución en el entorno que se creyere conveniente (p. 57). 
Para los autores, dicha investigación debe entender la psicología de los consumidores a la hora de preferir un producto mediante la publicidad. De tal manera, que las campañas se diseñen, bajo una lógica de creatividad, innovación y el mejoramiento de los bienes y servicios. Para que las empresas puedan determinar la demanda de ciertos bienes y servicios se auxilia de la investigación para recoger la información necesaria.

Un primer acercamiento partió de la prueba, error e investigación, para ver si determinado producto se vendía o no. Pero, generaba un problema en aquellas empresas grandes, ya que les podía acarrear pérdidas enormes. Así pues, las investigaciones de mercados fueron una herramienta útil para anticiparse en conocer los deseos y necesidades de los mercados, por ende, las fuentes para obtener información de una empresa, son los siguientes:

a. Datos de ventas de los productores.

b. Retiros de bodega y auditoría de datos.

c. Paneles de consumidores de registro diario.

d. Investigación específica por encuestas.

e. Mercados industriales o comerciales

Esta serie de elementos les permite a los investigadores tomar mejores decisiones sobre el desarrollo de nuevos productos y su lanzamiento al mercado. Establece una fase previa antes de comenzar la producción y ser eficaces en llevar los servicios que el cliente necesita. Como plantea Malhotra (2008), la investigación de mercados ofrece una serie de información importante a los investigadores sobre el marketing de diferentes productos que una determinada empresa desea lanzar al mercado.

Paraidentificar por quéla demanda deun producto cae en el mercado, las empresas se auxilian de los métodos y técnicas de investigación, sobre todo aquellas que no generen costos altos. Por ende, las técnicas cualitativas dan un gran aporte a las investigaciones de mercados. Las entrevistas personalizadas, entrevistas por teléfono y por correo son las más utilizadas por su bajo costo.

Por ello, la importancia de este tipo de investigaciones, en palabras de Hair, Bush y Ortinau (2009): "El propósito fundamental de la mercadotecnia es que las empresas planeen y ejecuten las actividades de fijación de precios, promoción y distribución de productos, servicios e ideas para generar intercambios que satisfagan a la empresa y a sus clientes (...)" (p.4). De tal manera que lo fundamental en todo proceso para estudiar la preferencia de los productos por un determinado estrato o estratos de consumo, deberán ser el objetivo del cual parte toda investigación.

Cuando se inicia una investigación hay que tomar en cuenta tres aspectos importantes:

- Localizar e identificar nuevas oportunidades de mercado para una compañía.

- Identificar grupos de clientes de un mercado que tengan necesidades, características o preferencias parecidas.

- Identificar las ventajas y desventajas de los competidores actuales y potenciales. 
Identificados los tres aspectos anteriores, los investigadores de mercados, definen los elementos del proceso de investigación, entre los cuales, según Malhotra (2008), pueden ser: definición del problema, desarrollo del enfoque del problema, formulación del diseño de investigación, trabajo de campo o recopilación de datos, preparación y análisis de datos y elaboración y presentación del informe. Aunque no todos coinciden en los mismos elementos, esta es la estructura básica que toda investigación debería llevar.

Tomando en cuenta lo anterior, Pilco y Ruíz (2015) y Pope (1984), mencionan que entre las principales técnicas de investigación de los estudios de mercado, se encuentran las entrevistas personales, por correo y por teléfono. Además, los tipos de muestra que se utilizan pueden ser probabilísticos y no probabilísticos, dentro de los cuales por el costo se utilizan los últimos. La aplicación de distintos métodos y técnicas dependerá de los objetivos que persiga el estudio y que se quiere conocer de la empresa en cuanto conocer la demanda de un producto o productos en el mercado.

A continuación, se expondrán las principales técnicas utilizadas por la investigación de mercados y con las cual coinciden la mayoría de investigadores expertos en esta área. Las entrevistas personales han sido una de las más utilizadas y tradicionales desde los comienzos, sobre todo porque estas permitían interactuar de manera directa con los entrevistados. Para seleccionar a los entrevistados, se pueden utilizar el muestreo probabilístico y no probabilístico, según se lo planteen los intereses de las empresas.
Para llevarlas a cabo se prepara una serie de preguntas, que se han considerado vitales a la hora de obtener la información de los entrevistados, por ende, algunas de estas preguntas claves para conocer las preferencias de los consumidores en el mercado, pueden ser las siguientes: ¿de qué tamaño es la demanda posible en el mercado objetivo? ¿Qué tan sensible es la demanda a los cambios de precios? ¿Qué factores, aparte del precio, les parecen importantes a los clientes? ¿Cuáles son los pronósticos de ventas en diversos niveles de precios? ¿Se identifican los consumidores con cierto tipo de marcas de la empresa? ¿Se sienten motivados los clientes con el lanzamiento de promociones a la hora de consumir los productos de una empresa?

Así pues, cada una de las preguntas que se formulen en el instrumento utilizado por los investigadores, será de mucha importancia para tomar decisiones o dar un giro en las empresas. Este tipo de técnica, puede ofrecer la diversidad de estratos de consumo en las diferentes poblaciones, según sea el nivel adquisitivo de las mismas, además que permitirá establecer los lineamientos de la publicidad de las empresas.

Otra de las técnicas utilizadas es la entrevista por teléfono, alternativa que representa para las empresas costos bajos en la realización de investigaciones con poblaciones dispersas y grandes. Su puesta en funcionamiento, se debió a los avances tecnológicos y a la flexibilidad que mostraba para los gerentes de las empresas. En sus inicios el procedimiento para llevarla a cabo se realizaba desde la casa de los investigadores, pero posteriormente se hizo en los centros de entrevistas que se encontraban en las mismas empresas, esto permitió tener un mayor control sobre las entrevistas realizadas. 
Además, las entrevistas por teléfono permiten una flexibilidad a la hora de escoger la muestra, ya que los investigadores pueden seleccionar del directorio telefónico a las personas que cumplan su interés de acuerdo a su dirección, lo cual facilita el trabajo. Algunas de las preguntas comunes utilizadas en este tipo de entrevistas son: ¿cada cuánto tiempo consume aquel tipo de productos? ¿Con qué color identifica alguna marca? ¿Ha observado algún tipo de publicidad en la TV de algún producto? ¿Seguiría consumiendo dicho producto si aumentara de precio? ¿Con qué tipo de imagen relaciona a esa marca? ¿Cuánto dinero gasta en consumir aquel producto? De esta manera, los entrevistadores pueden usar su creatividad a la hora de realizar este tipo de entrevistas, lo cual le ampliará la información sobre la mercadotecnia de ciertos productos.

Asimismo, se encuentra la entrevista por correo, aunque es de las técnicas más complicadas dentro de la investigación de mercados. Su proceso se inicia formulando un cuestionario o batería de preguntas; luego, se establece la muestra de la población a la cual se le enviará la entrevista. Muchos investigadores prefieren hacer uso de pruebas antes de hacer la investigación para detectar posibles errores. Los mercados que hacen uso de esta técnica son los industriales y los médicos, debido a la facilidad para hacer estudios en poblaciones grandes.

Algunos de los problemas que presenta esta técnica son: la falta de llenado del instrumento por parte de los entrevistados, el sesgo por los que no envían el formulario, límites a la hora de hacer las preguntas, falta de profundidad en los estudios, entre otros. De las tres técnicas mencionadas, esta es la menos utilizada por los mercadólogos o gerentes a la hora de querer conocer la preferencia de productos de una determinada empresa, por lo difícil de obtener resultados precisos, cuestión necesaria para los tomadores de decisiones.

A continuación, se presenta un ejemplo de preguntas enviado a los correos para hacer este tipo de estudios: ¿Cómo nos conoció? ¿Cuál es su grado de satisfacción con los productos/ servicios? ¿Recomendaría el producto a otras personas/empresas? ¿Con qué colores asocia nuestros productos? ¿Qué tipo de productos consume diariamente?

De esta manera, las tres técnicas integradas son muy importantes a la hora de hacer estudios de mercado, porque contribuyen a profundizar sobre las motivaciones que llevan a un cliente a elegir determinado producto de una empresa. Tal como lo explica Parrado (2013), la psicología juega un papel importante a la hora de que los consumidores prefieran un producto y no otro. Es decir, cómo modificar las conductas de los clientes usando la publicidad.

\section{Conclusiones}

La ciencia y la investigación juegan un papel importante, en el desarrollo histórico social de cualquier país, su propósito es llegar a las causas que provocan los fenómenos sociales y naturales, por medio de un método científico. En ese sentido, se clasifican en dos grandes polos que buscan interpretar la realidad de diferentes maneras.

La ciencia como un todo, pretende establecer leyes generales sobre la sociedad y la naturaleza que permita estudiar la realidad, mediante el 
establecimiento de hipótesis. Por ello, se ha visto influenciada por el desarrollo histórico y social, los cuales establecen influencia en la producción científica de una época.

Los métodos y técnicas de la investigación funcionan como dos auxiliares distintos a la hora de estudiar los fenómenos; en tal sentido, muestran las formas de cómo interpretar la realidad mediante la utilización de diferentes instrumentos, ya sean cualitativos o cuantitativos, ambos buscan la demostración de una verdad de distintas maneras.

Los métodos de investigación más utilizados por los investigadores son los de carácter cualitativo, que buscan penetrar en la subjetividad de las personas, los cuantitativos que pretenden medir y comprobar las hipótesis sobre ciertos fenómenos estudiados mediante fórmulas estadísticas y los pluralistas metodológicos que fusionan a los dos anteriores, ya que cualquiera presenta un carácter científico.

La investigación de mercados busca organizar, recopilar e interpretar datos sobre información que permita conocer las preferencias, nivel de consumo y motivaciones a la hora de comprar ciertos productos, lo cual le brinda a las empresas desarrollar nuevos productos en el mercado nacional e internacional.

El proceso mediante el cual, se realiza una investigación de mercados es casi igual al que se utiliza en cualquier investigación en las ciencias naturales o sociales, con la diferencia que su objeto de investigación son los clientes y el comportamiento de ciertos productos a la hora de ser preferidos por los consumidores en los mercados.
Las tres principales técnicas de investigación que se usan en los estudios de mercado son: las entrevistas o encuestas personales, las entrevistas o encuestas por teléfono y las entrevistas o encuestas por correo. Cada una de ellas, contribuye de diferente manera a la hora de obtener información sobre los clientes y productos de una empresa.

Un factor clave que interviene en los estudios de mercado es la psicología del consumidor, debido a que las motivaciones en los clientes es un factor clave para comprender si la publicidad está haciendo efecto en los mismos, llegar a los estratos diferentes de consumo significa un éxito para cualquier empresa.

\section{Referencias bibliográficas}

Benassini, M. (2009). Introducción a la investigación de mercados: enfoque para América Latina. México D.F.: PEARSON.

Danermark, B., Jakobsen, L., Ekstrom, M. y Karlsson, J. (2016). Explicando la sociedad. El realismo critico en las ciencias sociales. San Salvador, E1 Salvador: UCA Editores.

Dieterich, H. (1996). Nueva guía para la investigación cientifica. México D. F.: Editorial Planeta mexicana.

Hair,J.,Bush,R.,Ortinau,D.(2009).Investigación de mercados, en un ambiente de información digital. México D.F.: Mc Graw Hill.

Kédrov, M. y Spirkin, A. (1968). La ciencia. México D.F.: Editorial Grijalbo. 
Malhotra, N. (2008). Investigación de mercados. México D.F: PEARSON Educación.

Navas, V. (2014). La investigación social. Su metodología. San Salvador, El Salvador: Centro de Investigaciones en Ciencias y Humanidades.

Parrado, F. (2013). J. B. Watson y la publicidad, los inicios de la psicología del consumidor. Revista colombiana de Psicología, 22(2), 401-406.

Pilco, W., y Ruiz, L. (2015). La investigación de mercados como una disciplina estratégica. Riobamba, Ecuador: Escuela Superior Politécnica del Chimborazo. Instituto de Investigaciones.
Pope, J. (1984). Investigación de mercados. Guía maestra para el profesional. Colombia: Editorial Norma.

Rojas, R.(1996). Guía para realizar investigaciones sociales. México D.F: Plaza y Valdez Editores.

Rosental, M., e Iudin, P. (1946). Diccionario filosófico marxista. Montevideo, Uruguay: Editorial Pueblos Unidos.

Tamayo, M. (2006). El proceso de la investigación cientifica. México D.F.: LIMUSA. 